\title{
A Patient with COVID-19 Treated with ECMO for 26 Days
}

\author{
Chi Zhang, Bo Shi, Haichun Ma and Naifu Dong \\ Department of Anesthesia, The First Hospital of Jilin University, Changchun, Jilin, China
}

\begin{abstract}
In December 2019, an outbreak of a novel coronavirus disease 2019 (COVID-19) was reported in Wuhan, China, which was subsequently reported in other countries worldwide. COVID-19 is typically an acute self-limited disease that can rarely be fatal with a $5.6 \%$ case mortality rate (May, 2020), mainly due to substantial damage to pulmonary alveolar structures, and subsequent respiratory failure. Given the previous experience, extracorporeal membrane oxygenation (ECMO) has been proven to be an effective therapy in the treatment of respiratory failure or acute respiratory distress syndrome (ARDS). On the basis of similar principle, ECMO may also be an effective therapy in the treatment of severe COVID-19. However, due to huge cost, it is not common to apply ECMO continuously for a long time. We, herein, describe a COVID-19 pneumonia patient, a 77-year female \who was treated with ECMO for 26 days. The hospitalisation costs of the patient were highest in jilin Province.
\end{abstract}

Key Words: COVID-19, ECMO, Pneumonia.

How to cite this article: Zhang C, Shi B, Ma H, Dong N. A Patient with COVID-19 Treated with ECMO for 26 Days. J Coll Physicians Surg Pak 2020; 30(JCPSPCR):CR171-CR173.

\section{INTRODUCTION}

At present, much research is focused on how to improve the survival rates for patients with novel coronavirus disease 2019 (COVID-19), reduce complications and improve prognosis in the wake of prevailing pandemic. Although the application of extracorporeal membraneoxygenation (ECMO) as abridgeto rehabilitation is currently feasible, it has a high risk for morbidity and mortality. ${ }^{1}$ This case report attempts to present a cost-benefit analysis of ECMO therapy by describing the entire course of COVID-19 severe case treated with this form of therapy.

\section{CASE REPORT}

A 77-year female, with a fever of $38^{\circ} \mathrm{C}$, was admitted to the Department of Cardiology and then transferred to Intensive Care Unit (ICU) due to severe respiratory distress. CT chest showed viral pneumonia, and a throat swab test was positive for the severe acute respiratory distress syndrome coronavirus 2 (SARS-CoV-2) by real-time reverse transcriptase PCR assay, confirming that the patient had COVID-19-associated peumonia.

The medications used, their dosages and durations throughout the treatment process are shown in Figure 1.

Correspondence to: Dr. Naifu Dong, Department of Anesthesia, The First Hospital of Jilin University, Changchun, Jilin, China

E-mail: dongnaifu123@163.com

Received: May 23, 2020; Revised: November 10, 2020; Accepted: November 11, 2020

DOI: https://doi.org/10.29271/jcpsp.2020.JCPSPCR.CR171

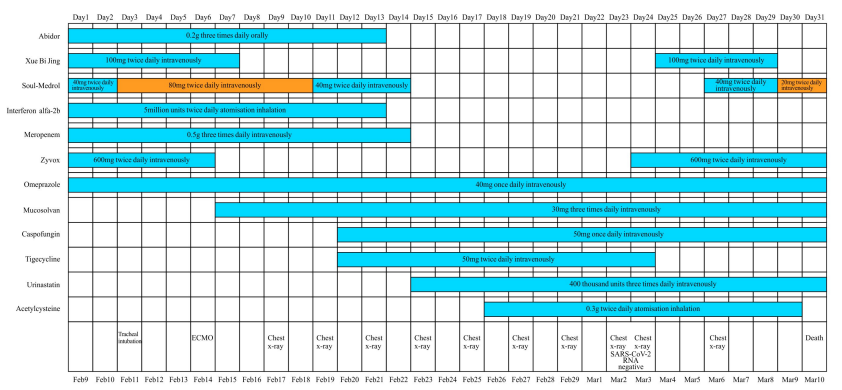

Figure 1: Timeline of disease course according to days from hospital admission. Note: The drugs used throughout the treatment, the dosage, and the tests performed.

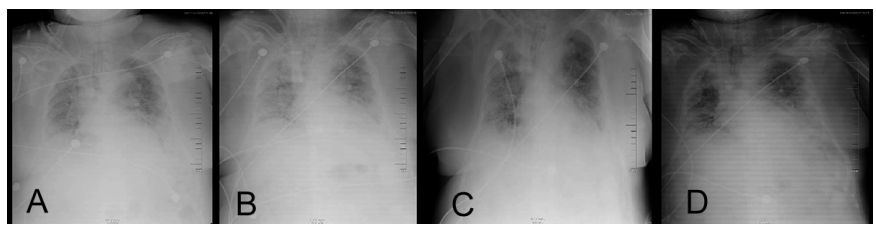

Figure 2: Changes of chestradiographs during treatment. (A)Chestradiograph on day 9 after admission; (B) Chest radiograph day 11 after admission; (C) Chest radiograph on the day 24 after admission; (D) Chest radiograph on the day 27 afteradmission.

At first, we used abidor, xuebijing and interferon alfa-2b as antiviral therapy, and methylprednisolone was administered to attenuate lung inflammation. Then, we added linezolid and meropenem to prevent secondary infection. But the patient did not improve after the above treatment. According to previous experience, ECMO was immediately applied, which was of great benefit to the patient. ${ }^{1}$ At the ninth day, the first chest X-ray after ECMO was taken and showed severe lung inflammation, as shown in Figure 2A. Two days later, we took the second chest $X$ ray, but, there was no improvement in lung inflammation and the inflammation was still severe, as shown in Figure 2B. After 
24 days of treatment, a throat swab test for the virus was done twice, and was negative. Chest X-ray showed that the lung inflammation of the patient was better than before, as shown in Figure 2 C/D. Despite the improvement in X-ray imaging, no improvement was found in the lung function of the patient, and he still could not be weaned off from ECMO support. Finally, the patient died of COVID-19 related complications after involvement of a lot of personnel and spending of numerous material resources.

\section{DISCUSSION}

Uptil now, no drug has been confirmed to be safe and effective for severe COVID-19 pneumonia. The World Health Organization interim guidelines made recommendations for the treatment of ARDS in this setting, including referring patients with refractory respiratory failure to expert centres capable of providing ECMO. ${ }^{2}$ ECMO therapy, as a bridge to rehabilitation, is feasible, albeit with an increased risk of morbidity and mortality. ${ }^{3}$ Support with ECMO is typically offered for the most critically ill patients in regions with the extensive resources required to provide this therapy. ECMO is not a frontline therapy, but one that is offered when all resources are stretched in a pandemic. Although studies have shown that new technologies can allow the management of patients for $>200$ days on ECMO without dying, ${ }^{4}$ it does not necessarily mean that we should offer it to every patient. The patient outcome would probably improve further, if the duration of ECMO could be shortened, thereby reducing the risks of complications. A reduction of the waiting time on ECMO could not only reduce complications directly related to ECMO, such as bleeding and stroke, but also those indirectly related, such as critical illness myopathy and infections. ${ }^{5}$

In the retrospective study conducted by Yang et al., 52 critically ill adult patients were identified with SARS-CoV-2 pneumonia and were admitted to ICU; among them, 31 patients died at 28 days, six patients received ECMO, and five of them died and one patient was still on ECMO at the endpoint. ${ }^{6}$ Another retrospective study conducted by Zhang et al. included 221 patients with laboratory confirmed SARS-CoV-2 pneumonia, 48 patients with severe patients developed ARDS, and 10 of them received invasive mechanical ventilation (IMV) and ECMO support.

Among those, two patients showed clinical benefit and were discharged while three did not survive.

The rest five patients were still on ECMO at the endpoint. ${ }^{7}$ Given lack of randomised clinical trial of ECMO in COVID-19, we could not conclude whether SARS-CoV-2-infected patients have benefited from ECMO at this time.

The role of ECMO in the management of COVID-19 is unclear at this point. However, ECMO is one of the treatment options in severe ARDS. ${ }^{8}$ ECMO is an invasive procedure with significant potential for complications, and the high costs could present economic challenges to the health system. ${ }^{9}$ Clinical judgment is needed to decide whether ECMO is effective or not, accompanied by understanding the risk-to-benefit ratio.
Finally, the patient died of COVID-19 related complications after involvement of a lot of personnel and spending of numerous material resources. Therefore, the role and usefulness of ECMO in COVID-19 patients still needs to be explored further.

\section{CONFLICT OF INTEREST:}

Authors declared no conflict of interest.

\section{PATIENT'S CONSENT:}

A written informed consent was obtained from the patient.

\section{AUTHORS' CONTRIBUTION:}

CZ: Substantial contributions to the conception or design of the work; or the acquisition, analysis, or interpretation of data for the work.

BS: Drafting the work or revising it critically for important intellectual content.

HM: Final approval of the version to be published.

ND: Agreement to be accountable for all aspects of the work in ensuring that questions related. to the accuracy or integrity of any part of the work are appropriately investigated and resolved.

\section{REFERENCES}

1. Lee JJ, Hwang SM, Ko JH. Efficacy of veno-venous extracorporeal membrane oxygenation in severe acute respiratory failure. Yonsei Med J 2015; 56(1):212-9. doi: 10.3349/ymj.2015.56.1.212.

2. World Health Organization. Clinical management of severeacute respiratory infection when novel coronavirus (2019-nCoV) infection is suspected - interim guidance. Published January 28, 2020. Accessed February 11, 2020.

3. Toyoda Y, Bhama JK, Shigemura N, Zaldonis D, Pilewski J, Crespo M, et al. J Thorac Cardiovasc Surg 2013; 145(4): 1065-70. doi: 10.1016/j.jtcvs.2012.12.067.

4. Skansebo E, Broomé M, Magnusson J, Riise GC, Dellgren G. Extended use of extra corporeal membrane oxygenation as bridge to lung transplantation in two patients. Eur J Cardiothorac Surg 2020; 15(1):16. doi: 10.1186/s13019020-1046-0.

5. Dellgren G, Riise GC, Swärd K, Gilljam M, Rexius H, Liden $\mathrm{H}$, et al. Extracorporeal membrane oxygenation as a bridge to lung transplantation: A long-term study. Eur J Cardiothorac Surg 2015; 47(1):95-100. doi: 10.1093/ ejcts/ezu112.

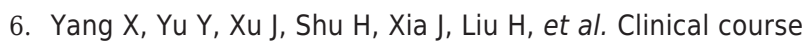
and outcomes of critically ill patients with SARS-CoV-2 pneumonia in Wuhan, China: A single-centered, retrospective, observational study. Lancet Respir Med 2020; 8(5):475-81. doi: 10.1016/S2213-2600(20)30079-5.

7. Zhang G, Hu C, Luo L, Fang F, Chen Y, Li J, et al. Clinical features and short-term outcomes of 221 patients with COVID-19 in Wuhan, China. J Clin Virol 2020; 127:104364. doi: 10.1016/j.jcv.2020.104364.

8. Brodie D, Slutsky AS, Combes A. Extracorporeal life support for adults with respiratory failure and related indications: $A$ review. JAMA 2019; 322(6):557-68. doi: 10.1001/jama. 2019.9302. 
9. Maclaren G, Fisher D, Brodie D. Preparing for the most critically III patients with COVID-19: The potential role of extracorporeal membrane oxygenation. JAMA 2020; 323(13):1245-6.doi.org/10.1001/jama.2020.2342. 